\title{
The role of the psychiatrist in the general hospital*
}

\author{
A. H. CRISP \\ M.D., M.R.C.P.E., D.P.M. \\ Professor of Psychiatry, \\ St George's Hospital Medical School, Hyde Park Corner, London, S.W.1
}

The CURRENT trend for the National Health Psychiatric Service is to integrate it into the general hospital system. More psychiatrists are spending more time doing clinics in general hospitals. More have some or all of their beds in general hospitals. The overall advantages and disadvantages of this state of affairs have recently been propounded elsewhere, both in the U.K. (McKeown, 1966 ; R.M.P.A. 1967 Memorandum) and in the U.S.A. (Kaufman, 1965). From the standpoint of the status of mental illness and psychiatry and from the possible standpoint that all illness, whether presenting socially, psychologically or somatically, is really 'psychosomatic' illness meriting the collective attention of doctors from many disciplines, such amalgamation is regarded by some as a progressive move. At the same time it presents problems of special management of predominantly mentally and socially ill patients-to do with their restraint on the one hand, their acting out on the other, in the intense and crowded milieu of a busy general hospital; behaviour which may intensify the rejection which is sometimes the lot of the mentally ill patient. Furthermore, the opportunities for a fully active therapeutic community within the psychiatric unit are probably less when not backed by the resources of a spacious and independent psychiatric hospital.

The bringing together of psychiatric services with other medical services can also lead to other situations. Firstly, the involvement of the psychiatrist in an increasing load of patients with fairly obvious neurotic problems but who have in the past been carried in general hospitals in one clinic after another being systematically investigated, largely with negative results, over the years. Secondly, the involvement, to a greater or lesser extent mainly according to the psychi-

\footnotetext{
*Paper read at Quarterly Meeting of Royal MedicoPsychological Association, February 1967. This study was conducted while the author was working as Senior Lecturer in the Academic Department of Psychiatry, Middlesex Hospital, London, W.1.
}

atrist's inclination, in the broader field of illhealth, probably mainly that section presenting to physicians, in which there is no evidence of formal mental illness but in which it may be that stress factors, having to do with the patient's personality, life experience and life situation, are contributing, in a major or minor way, to the patient's disease. It is these two points which I wish to concentrate on in this paper.

Firstly, however, there are several recent reports in the literature of studies of the incidence of mental illness in some sections of the general hospital population and of the use made of psychiatric services in general hospitals. In 1964 Davies surveyed studies reported on in the field of psychiatric illness in general hospitals. He drew on eleven studies, of which only two had been done in the United Kingdom, and which reported incidences of between $12 \%$ and $52 \%$ for psychiatric illness presenting in medical outpatient clinics. The mean over the eleven studies was $27.3 \%$. The investigators were usually referring to straightforward psychiatric illness and they went on to find a further $10-40 \%$ of patients, in the different studies, who presented with concurrent 'organic' and psychiatric illness. Davies was unable to find any comparable studies in the field of surgical out-patient clinics but recalls Macy \& Allen's study reported from the Mayo Clinic in 1933. For 5 years they followed 235 patients diagnosed as having neurotic ill-health by the clinic. During this time 200 of these patients had 289 separate operations (mainly tonsillectomy and gynaecological operations) which were only rarely followed by symptom relief. Davies also recalls that in 1939 Ziegler found 111 depressive patients who had first attended a surgeon with complaints of fatigue, loss of appetite, weight loss, insomnia and unusual bodily sensations.

More recently Priest (1962) reported on his analysis of 1000 consecutive female and male medical out-patient referrals to a provincial English general hospital in terms of the final diagnosis applied to them and found that $16 \%$ 
were thus classified as having clear-cut psychoneurotic illness.

On another tack Bryson had in 1945 expressed the view that $60 \%$ of gynaecological patients needed some psychiatric understanding-a finding borne out by Culpan, Davies \& Oppenheim (1960) in their own study of gynaecological outpatients in which they had used the C.M.I. (Cornell Medical Index) to obtain a measure of psychological disability.

Davies, in his 1964 review, went on to present his own findings amongst 100 consecutive female medical out-patients (patients took part in a halfhour interview and were not told that they were being examined by a psychiatrist). In his judgment and using the C.M.I. as an aid he found that forty-three of the patients had 'organic' illness only, thirty-eight had psychiatric illness only and thirteen had both psychiatric and 'organic' illness-a group he designated as 'mixed'. It was not possible to make discrete diagnoses in six patients. Patients in the 'mixed' group were considered to show definite psychiatric illness and this group did not include patients with such possibly psychosomatic disorders as peptic ulcer, asthma, etc., but in whom there was no evidence of classical psychiatric disorder. Nevertheless, $51 \%$ of female patients attending a medical outpatient clinic were found to have formal psychiatric illness.

Davies estimated that roughly one-third of these $51 \%$ might be helped by simple reassurance, another third by further support and that the remaining third needed full psychiatric investigation (in fact $18 \%$ of patients attending a female medical out-patient clinic). With this actual disposal Davies later judged that over $50 \%$ of these patients were recovered 3-6 months later.

He goes on to describe the lower incidence$5 \%$ of psychiatrically ill only and $16 \%$ 'mixed' -found by his co-worker Culpan in a male medical clinic ( $21 \%$ incidence of psychiatric illness). He concludes by recommending the C.M.I. as a useful tool for physicians wishing to detect psychiatric illness in their patients. He also emphasizes the need to approach patients as individuals with social, interpersonal and personal problems, if we are to further our understanding of illness.

In 1965 Maclay reported a similar study in a medical out-patient clinic but without using the C.M.I. It is noteworthy that she did not find a sex difference in the incidence of psychiatric illness in this population. However, her findings are not otherwise dissimilar. Of 100 patients (every third one attending the clinic) forty-five were found by the physician after full investigation to have a 'structural basis for their symptoms'. In the other fifty-five all investigations were negative. Twenty-two of this latter group were under the age of 30 as distinct from three in the former group. Twenty-one of the latter group were judged by Maclay, without her having any knowledge of the physician's findings, to have formal mental illness, as against five in the former group (i.e. $26 \%$ of the whole out-patient group). She did not include personality disorders or the psychosomatic groups in this figure. The younger ages of the group in which physical investigations were negative is of interest here. It would be interesting if these patients, regarded as formally mentally ill now and found to have no 'structural' basis for their symptoms, were found to become the psychosomatic group of the future-when tissue breakdown and macroscopic disease has developed, so that they then shift to the 'structurally disordered' department.

If we allow, for a moment, the "either organic or functional' view to prevail then the size of 'mixed' groups in these two studies is again of interest and is reminiscent of two other studies. In 1960 Herridge reported his observation that $5 \%$ of over 200 psychiatric in-patients were found to have major physical illness-mainly presenting with organic mental syndromes. In 1961 Jacobs \& Ritchie Russell reported that they had found $6 \%$ of neurological out-patients followed for 1-6 years and initially diagnosed as 'functional' to have developed neurological disease to account for their presenting symptoms.

The above reports of the incidence of psychiatric illness, largely in medical out-patient populations, are of studies which have been done by the psychiatrists entering the general hospitals and attendant clinics specifically so as to conduct their investigations. In 1963 Kenyon \& Rutter reported on 21 months' experience during which time a full-time registrar in psychiatry was available for psychiatric referrals in a general hospital. During this time 319 patients were referred, two-thirds as in-patients and one-third from out-patient departments: $62.5 \%$ were referred from medical departments (including paediatrics, dermatology and neurology), $12.9 \%$ were referred from surgical departments and 9.7\% from obstetric and gynaecological departments. Over half of these were diagnosed by the psychiatrists concerned as suffering with affective disorder. $20 \%$ of the total patient group were admitted to a psychiatric hospital following the referrals. In their discussion the authors make a point which psychiatrists and physicians often 
seem concerned to express: namely 'there is no place for the narrow specialist (psychiatrist) hiding behind jargon and elaborate dynamic psychopathology'. On the other hand they hint that some attempt to understand and communicate the psychopathology may be justified when they comment 'the psychiatrist must be prepared to offer a firm opinion often in the face of a certain amount of scepticism and in a language that all can understand'. It is also clear in their report that they frequently call upon the services of the almoner, now called the medical social worker, in their efforts to help patients referred to them.

In 1962 Fleminger \& Mallett reported on psychiatric referrals from medical and surgical wards to a psychiatric department established within the walls of the general hospital and able to provide a service. They reported on data accumulated over a 5-year period. Their salient findings were: (1) $0.7 \%$ of 50,000 admissions (one in 140) of patients were referred for psychiatric opinion; (2) $80 \%$ were referred by physicians; $20 \%$ were referred by surgeons and gynaecologists ; (3) $40 \%$ of patients seen had no organic disease; (4) nearly one-third of referrals were for aid in assessment of bodily complaints ; (5) almost half were referred on account of overt mental disorder; (6) there was a high incidence of hysteria but depression was commonest; (7) $5 \%$ had toxic or other types of confusional state; (8) one-third were advised to attend psychiatric out-patients; (9) $23 \%$ were transferred directly to a psychiatric bed; and (10) $1 \%$ required compulsory admissions. More recently Brough \& Fleminger (1965) have reported on out-patient psychiatric referrals from other hospital out-patient clinics and with roughly similar findings to those reported in their in-patient referral study. However, there was a lower incidence of hysteria and organic reaction and a higher incidence of anxiety state amongst the out-patient referrals whose overall referral rate was less than half the in-patient referral rate. They suggested that the smaller numbers referred by surgeons might be due to the shorter duration of stay of surgical patients (and therefore the less chance for disturbances of behaviour to unfold and present) as well as the more usual proposition that the physicians deal more commonly with illness having a psychological component. It is also the case that surgeons are even more closely focused down onto specific organs at a time when the patient is also in the same state of mind. They suggested that the low referral rate from the gynaecologists might be due to the latters' acceptance of emotional disturbance in relation to menstruation, pregnancy, etc., as often normal. Certainly it is common.

In 1966 Bridges, Koller \& Wheeler reported on the referrals to a psychiatric department within another London teaching hospital. They found that internal sources of referral accounted for 584 new patients seen during the year and that this number was two-thirds of the total new patients seen in the department during the year. Two hundred and four patients were referred from other wards with a diagnosis of attempted suicide and the authors comment on this high incidence and also on the high incidence of other diagnoses such as neurotic depression and personality disorder. These latter patients had usually presented to other departments with physical complaints.

Despite these interesting reports, however, there is nowhere in this country where a liaison service has been developed between physician, surgeon and psychiatrist in the way that this has been done in Rochester in New York State in the department run by Professor Romano and Professor Engel. For the last 20 years Professor Engel has built up a liaison service at present manned by eleven full-time senior staff and six trainee staff whose sole role is to examine the psychological in relation to the somatic aspects of disease as it presents in the wards of the hospital. These doctors are attached full-time to a ward and may themselves be primarily psychiatrists or physicians but always with a training in psychiatry and psychosomatics. The view of this department is that the majority of diseases occur or present to the doctor at a time of 'stress' in the patient's life which has its roots in the patient's personality and life situation and which merits attention being directed at its psychological aspects. Engel himself has expressed the view that the role of brain and mind in regulation of somatic processes and organismal adjustment will prove to be the most important basic discipline in the second half of the twentieth century'. The differences in the American and British approach to this field are shown by the following: in Fleminger \& Mallett's (1962) study the majority of in-patient referrals in a London general hospital were consequent on the appearance of overt psychiatric symptoms in the patient or else followed a series of investigations which had failed to reveal 'organic' pathology for complaints of bodily symptoms. On the other hand, Richmond (1965), defining the criteria for referral in an established liaison service in a general hospital in New York State, teaches that the physician himself will have made some 
initial appraisal of the value of psychotherapy and will also be aware of the existence of 'transference' and countertransference problems and when to seek psychiatric advice concerning them.

Whether or not we agree with the American view the conclusion from the sum total of the above studies must support the B.M.A. Committee's view expressed back in 1941 and referred to by Davies (1964) that, so far as patients in this country are concerned, "in any group of sick people something like $30 \%$ will be found to be suffering from conditions about which it is helpful to have psychiatric advice'-a view expressed before the effective advent of antibiotics and even more widespread immunization had shifted the pattern of ill-health even further in the direction of disorders of constitution and all that the term constitution, with its experiential as well as its genetic origins, implies.

\section{Present study}

This report is now concerned with my own experience over the last 5 years working as a psychiatrist employed in an academic department. The bed complement comprises fifteen psychiatric beds in a conventional ward within the Middlesex Hospital (a large general hospital and teaching hospital) and a further fifteen psychiatric beds at St Luke's-Woodside Hospital, a seventy-bedded psychiatric hospital annex about 6 miles away. The department was established just over 5 years ago, in parallel with an existing Department of Psychological Medicine based mainly at St Luke's-Woodside Hospital, with a principal intention of developing the teaching of psychiatry to medical students. This latter de- velopment has recently been described elsewhere (Hill \& Hinton, 1966).

The department was initially staffed by a professor, a senior lecturer, a lecturer and two senior house officers. The staffing has gradually increased so that now, on the medical side, there is a professor, two senior lecturers, a lecturer, a senior registrar, a registrar and three senior house officers, three part-time senior lecturers concerned almost only with teaching and a number of research workers. The clinical load, especially so far as seeing new patients is concerned, falls mainly upon six people, three of whom have consultant status. The academic staff needs to devote about $2 \frac{1}{2}$ days or five sessions per week to clinical work.

New patients, referred either from outside sources or other out-patient clinics in the hospital are seen in regular psychiatric out-patient teaching clinics within the department. New patients referred from other wards (i.e. inpatient referrals) are seen in addition as required. In this latter connection, just over 4 years ago a liaison service was established in which a number of members, both of the academic and clinical departments, were attached specifically to certain medical or surgical wards, usually thereby each providing a psychiatric service to two physicans or two surgeons. Another intimate link has been slowly forged since the students who passed through the psychiatric firm (a 3month full-time appointment) 2, 3, 4 and 5 years ago now form part of the junior staff of the hospital both in our own department and in other departments throughout the hospital.

With such links in mind Table 1 shows the

TABLE 1

Sources of referral of all new patients including ward referrals to psychiatric department over a 5-year period

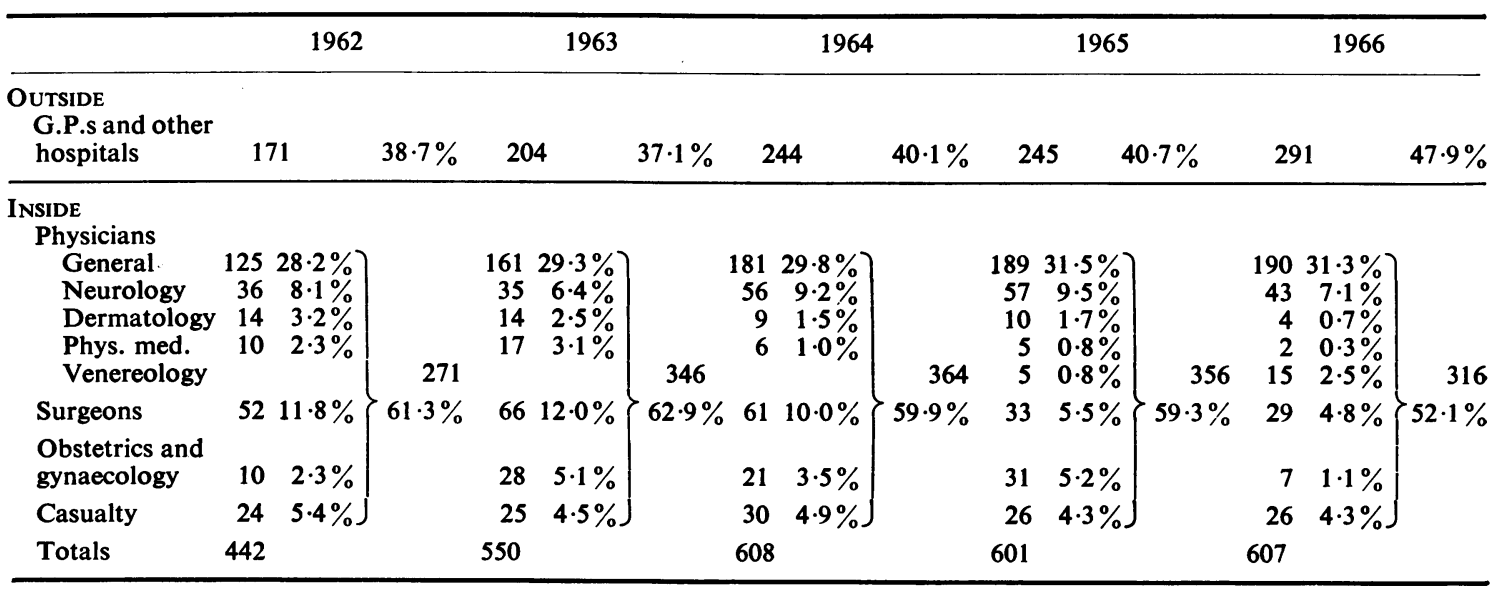


sources of referral of all new patients to the department over the last 5 years. More than half the new patients are referred from within the hospital, either from other out-patient clinics or as in-patient referrals. $30 \%$ of the total referrals, nearly 200 per year, are referred by general physicians and this figure has remained constant. The general physicians see between 4000 and 5000 new patients in each year. For instance, in 1965 they saw 4533 new patients and referred 189 patients (or $4.2 \%$ of their new patient total) as new patients to the psychiatric department. It must be borne in mind that there is another department of psychological medicine in the hospital which sees approximately the same number of new patients each year including a number referred from the physicians. Referrals from the neurologists account for 6$9 \%$ of all referrals to the psychiatric department and represent $4-6 \%$ of the new patients seen by the neurologists in any one year. It is interesting to note that referrals from the surgeons have fallen off and this has occurred in relation to the weakening of a surgical liaison link and the retirement of one surgeon who had referred numbers of patients. Previously referrals had been running at the rate of about $2 \%$ of new patients seen by the surgeons. Referrals from the obstetricians and gynaecologists have fallen dramatically and possibly in relation to the reluctance we have recently shown for recommending termination of pregnancy on psychiatric grounds. The numbers referred from Casualty have remained strikingly constant.

One consequence of all this is that we see a large number of new patients who have initially presented to other hospital specialists with complaints of bodily symptoms. The great majority of such patients referred from other out-patient clinics in the hospital have been found to have affective illness, depression, anxiety, tension or states of unresolved emotional conflict leaving them feeling inwardly anguished or angry and with associated somatic symptoms which have been the focus of their attention and complaint.

I now want to concentrate on my own liaison link, over the last 4 years, with two general physicians with beds on one male ward (eighteen beds) and one female ward (twenty beds).

Both these physicians and their registrars are in the habit of and capable of taking psychological and social factors into account when arriving at a diagnosis. They sometimes deal with such aspects themselves and may also, for instance, turn directly to the medical social workers as a source of social or psychological help for the patient or they may prescribe psychotropic drugs for the patient. Patients referred for a formal psychiatric opinion therefore tend to be either those admitted as psychiatric emergencies, those with psychological aspects or basis to their illness which have already been partially identified, or those with psychosomatic disorders, characteristically denying that they have an emotional problem but in whom one is suspected.

Table 2 shows the incidence of such referrals. These referrals, (307 in 5 years, including 239 in the last 3 years) were all formal referrals and do not include patients about whom there has been some passing discussion but no full evaluation and recommendation. The distinction made between psychiatric, psychosomatic and somatic is not one, in my view, that is always readily justifiable but, in using it here, I am referring to mental illness, psychopathy and subnormality as psychiatric illness, disorders such as ulcerative colitis, peptic ulcer, asthma, diabetes mellitus, essential hypertension, obesity and anorexia nervosa as psychosomatic illness and disorders such as cancer, meningitis, rheumatic heart disease, congenital malformation as somatic illness. With such a classification it can be seen that about one quarter of the patients fall into the psychosomatic category. A final category of 'mixed' diagnoses includes patients with both psychiatric and psychosomatic or somatic illness.

Overall, once the liaison link had become established, approximately $10 \%$ of all admissions (i.e. about eighty out of 800 per year) were referred annually for a psychiatric opinion.

Table 3 shows the reasons for admission to the medical wards of the 307 patients on whom this psychiatric epinion was sought over the 5 years. The majority were admitted for investigation of such symptoms as fatigue, dizziness, faints, vomiting, diarrhoea, polydipsia, weight loss, pain, hirsuties, amenorrhoea, etc. Such patients were admitted with only a differential diagnosis, usually including a possible psychiatric diagnosis, and with the knowledge that a psychiatric service for further evaluation and disposal was available. Most of the group admitted with psychiatric illness were individuals who had attempted suicide.

Table 4 shows an attempt to categorize the 307 patients according to more specific final diagnoses and partial diagnoses. Nearly one fifth of the patients were considered to have a definite diagnosis of depression. Patients who had attempted suicide were only diagnosed as also having depression if this was quite definite. Many such patients did not show clear or pervasive features of depression but presented features of 
A. H. Crisp

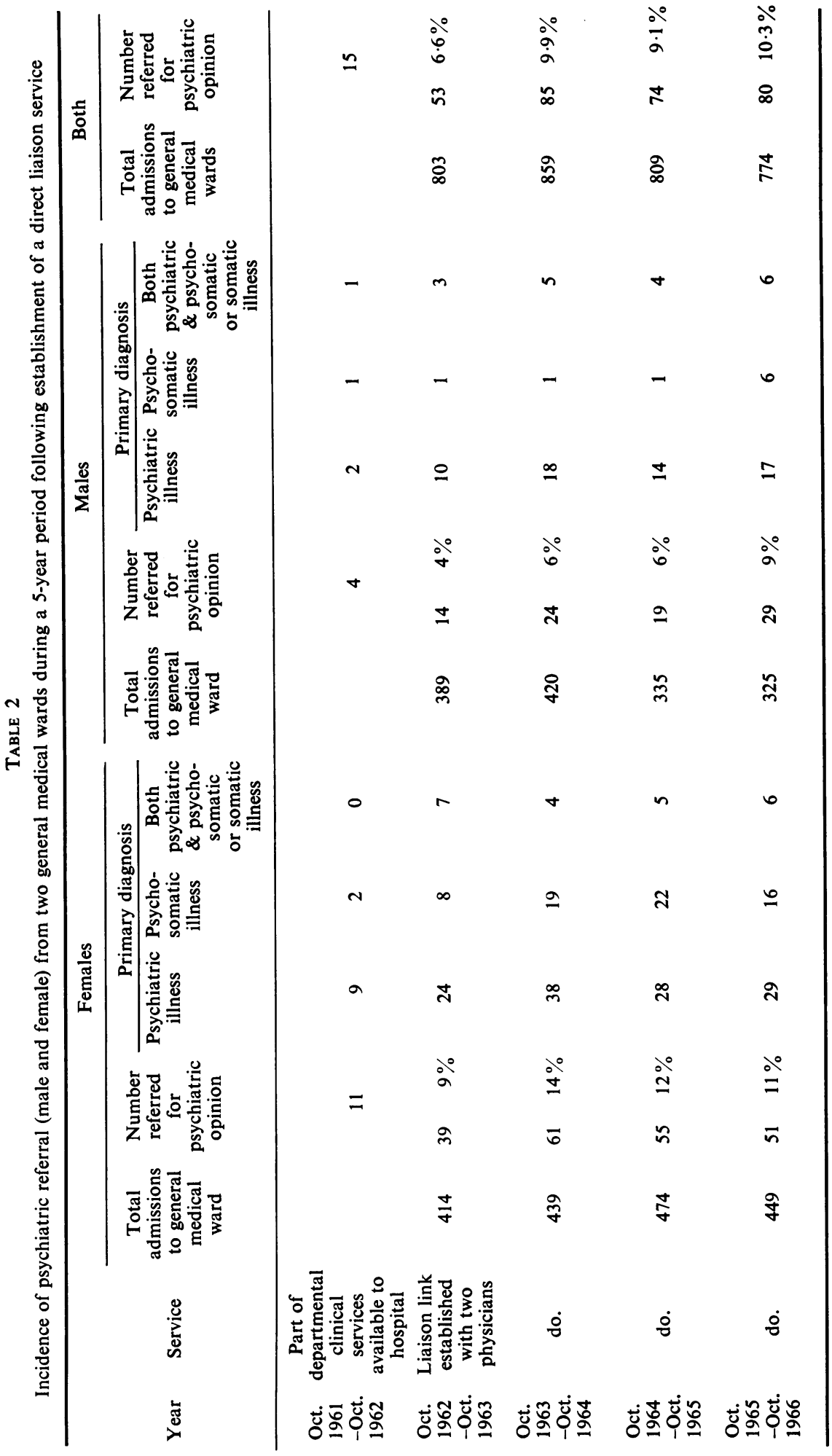

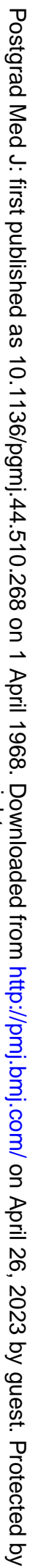


TABLE 3

Reasons for admission to two general medical wards (one male, one female) of 307 patients subsequently referred for psychiatric opinion

\begin{tabular}{lccccc}
\hline \multicolumn{1}{c}{ Reasons for admission } & $\begin{array}{l}\text { Oct. 1961- } \\
\text { Oct. 1962 }\end{array}$ & $\begin{array}{l}\text { Oct. 1962- } \\
\text { Oct. 1963 }\end{array}$ & $\begin{array}{l}\text { Oct. 1963- } \\
\text { Oct. 1964 }\end{array}$ & $\begin{array}{l}\text { Oct. 1964- } \\
\text { Oct. 1965 }\end{array}$ & $\begin{array}{l}\text { Oct. 1965- } \\
\text { Oct. 1966 }\end{array}$ \\
\hline $\begin{array}{l}\text { For further evaluation and treatment } \\
\text { (medical diagnosis established) }\end{array}$ & 2 & 14 & 23 & 15 & 18 \\
$\begin{array}{l}\text { For investigation of physical complaints-fatigue, dizzi- } \\
\text { ness, polyuria, vomiting, weight loss, pain, etc. }\end{array}$ & 12 & 36 & 46 & 40 & 51 \\
$\quad$ (medical diagnosis not established) & 1 & 3 & 16 & 19 & 11 \\
Psychiatric illness (including attempted suicide) & 15 & 53 & 85 & 74 & 80 \\
Totals & 1 & & & \\
\hline
\end{tabular}

TABLE 4

Three hundred and forty-eight diagnoses and partial diagnoses following psychiatric assessment in 307 patients referred from two general medical wards (one male, one female) during years October 1961 to October 1966

Depression (excluding attempted suicide)

Obsessional neurosis

Anxiety state

Anxiety phobic state

Conversion hysteria

Attempted suicide

Schizophrenia

Subnormality

Organic mental state

Addiction (including alcohol)

Homosexual conflict

Psychogenic somatic complaints

Personality disorder

Disorders of weight-anorexia nervosa -obesity

Essential hypertension

Rheumatoid arthritis

Ulcerative colitis

Peptic ulcer

Diabetes mellitus

Asthma

Others

personality disorder, addiction, homosexual conflict, anxiety state, etc., in which depression was regarded only as one aspect. Depression was considered a diagnostic entity when there were definite and more or less persistent features of sadness and self-reproach, whether or not immediately apparent, accompanied by reduced activity or, occasionally, agitation. Nearly all these patients had been admitted to hospital with physical complaints of the kind already described but were considered to be clearly closer to and capable of adopting a depressive posture rather than any other affective state. Patients with anxiety states and anxiety phobic states had often presented with symptoms of dizziness, headache, faints or palpitations. Hy. sterical conversion symptoms included amnesias, paralyses, anaesthesias, backache, deafness and dysphasia. The relatively high incidence of such phenomena in the present investigations accords with Fleminger \& Mallett's (1962) findings. The schizophrenic group had mostly been admitted either in stupor or else for the investigation of physical complaints of a delusional kind. The group of psychogenic somatic complaints comprises patients in whom there was a predominant complaint of pain or preoccupation with health and who were considered to have a psychopathological basis but without clear evidence of an underlying or alternative posture of depression or other affective illness. That there are twenty-six patients with anorexia nervosa in the list is a reflection, in my view, of the common occurrence of this disorder. I have described elsewhere some of the ways in which it may present and masquerade in medical wards (Crisp, 1967).

The final list of psychosomatic disorders presents a special problem with such patients prone to exercise extreme denial. They may seek immediate reassurance that the suggestion that emotional factors play a part in this illness does not imply that they behave neurotically or that the disease is in their 'imagination'. Sloane (1963) has recently written thoughtfully and extensively on this point. Such patients usually need, so far as $I$ am concerned, more time to be spent in the initial diagnostic interview. After 40-60 min one may have only the barest bones of a psychiatric history, minimal clues as to any pertinent area of relevant psychopathology and interpersonal conflict and still limited rapport with the patient. Only after an hour or more, may the patient find they have already committed themselves sufficiently to feel able, if the rapport has improved, to disclose themselves or 
others emotionally involved with them. Such patients may still find the process humiliating and threatening in the extreme. They are used to battling on, whatever their personal anguish and they may feel more ashamed than some neurotically disturbed patients at having disclosed a husband's or parent's 'weaknesses'. The dangers of concomitant or alternative depressive illness developing are often present and require special experience and judgment as to whether further exploration is justified or whether the psychiatrist is wiser to cease enquiry, collude in the patient's rapidly restoreable ability to deny emotional difficulties, and to recommend, for example, supportive treatment.

From early on it became apparent that the psychiatric report, often fairly lengthy, was more useful if it was typed. It became the custom to provide two copies, one for the medical notes and one for the general practitioner.

Ultimate recommendation in the 307 patients was as follows. In $35 \%$ of all cases the patient was seen once or twice only and the relatives probably seen once (either by the physician, his staff or myself). Advice concerning such patients was usually in the direction of prescribing some psychotropic drug, usually an antidepressant or tranquillizing one. In some of this $35 \%$ other treatment such as psychotherapy was recommended either as an immediate or second measure should they fail to improve with symptomatic treatment. However, this recommendation was made to the general practitioner either because the patient came from some other part of the country or because at that time no appropriate psychotherapeutic time was available in the department in the foreseeable future. Because of the usual limited availability of psychotherapy, recommendations that an attempt should be made to provide it were only made when such treatment was considered likely to really help the patient.

Some of the same group of patients were suffering with chronic and intractable neurotic disorders, e.g. life-long severe hypochondriasis or chronic conversion hysteria. Such patients frequently make heavy demands on conscientious doctors concerned not to miss the inevitable development of macroscopic bodily disease when it does come. At the most it was usually only possible to make suggestions as to how best to support, help with and cater for such patients' current interpersonal and social problems and often insatiable dependency needs which appeared to be mobilizing the current symptomatology, and to heave a sigh of relief that one didn't have to treat the patient oneself.
A further $6 \%$ of patients were seen and referred to other psychiatrists or psychiatric hospital. The majority of these were already waiting to see the other psychiatrist at the time that they were admitted into the medical bed; a few were transferred following compulsory detention. A further $3 \%$ of the patients refused to see a psychiatrist again; occasionally such patients hinted that they would be prepared to receive symptomatic treatment, such as supportive psychotherapy or psychotropic drugs, from their general practitioners and such recommendations were made if considered appropriate. Overall, therefore, $44 \%$ of the total patient group were not taken on for treatment in the department.

Of the remaining $56 \%, 13 \%$ were treated in the medical ward. Such treatment included drug treatment, training in relaxation, reassurance, occasionally brief individual psychotherapy and, slightly more often, two or three sessions of joint psychotherapy with the patient and spouse. $22 \%$ were taken on in a similar way but as outpatients referred to the psychiatric department from the start. $17 \%$ were transferred as inpatients to the psychiatric ward. The presence of psychiatric beds in the general hospital has been important for determining the type of $\vec{\bullet}$ patients treated in this way. For instance, it has $\underset{\infty}{\infty}$ been possible to treat patients with grossly un stable diabetes, the instability based in neurotic problems, and recurrently complicated by comas of diabetic, hypoglycaemic and hysterical origins with the patient in a psychiatric bed but also under readily available supervision by the physicians. In contrast is a final $4 \%$ of patients for whom, after an initial diagnostic interview in which a good relationship had seemed to be established (and only if this was so) a recommendation was made that they be discharged from hospital. At the same time they were given the departmental telephone number and invited to contact me if ever they really needed to. This group was almost entirely composed of patients who had attempted suicide. They were ambivalent about or resistant to admission to a psychiatric unit and beds were not always available. It was judged that compulsory detention was not the answer since they were likely to be discharged or to discharge themselves after 3 days and to be thereafter even more resistant to help. They were judged not to be clearly deter mined to kill themselves in the immediate future. They often had no current stable social position or interpersonal relationships. They rarely had a general practitioner. Two such patients subsequently took up the invitation and were taken into treatment. Three other such patients took 
the trouble to write 6-12 months later saying that they now considered themselves to be better and how helpful and supportive they had found the open invitation and how they had often been on the brink of telephoning. The remaining seven patients making up this category have not been heard of since discharge. Such measures as these are clearly unsatisfactory but sometimes seemed to be forced on one. Their failure or success is likely to depend at least to some extent on the quality of the relationship established during the single interview.

\section{Discussion}

In looking at the results of the present study it is evident that a specific liaison link with one or two physicians and with weekly contact is probably a major factor determining the extent of referral of patients for psychiatric opinion. The clinical psychiatrist is, therefore, in a position to influence the amount of such work he is called upon to do, depending upon his available time and inclination. It is also clear that the physicians in medical out-patient clinics referred nothing like the $20-50 \%$ of patients found by the other investigators to be presenting with psychiatric illness to such clinics. If we assume there was, nevertheless, a similar incidence in the present clinics then it may be that the physicians referred such patients back to their general practitioners, referred them round to other non-psychiatric clinics or else treated the patients themselves, either continuing to treat or attend to the presenting complaint of bodily symptoms eventually with occasional follow-up or else treating the patient's mental state, more often than not with psychotropic drugs. It would certainly have been quite impossible for even a small fraction of these patients, if they exist, to be seen in the psychiatric department in its present form. This brings me to a final point. It may well increasingly fall to the psychiatrist to contribute to the treatment of the so-called psychosomatic disorders. There is a great deal of energy being devoted at present to the proposition that some psychiatric illnesses have a distinct and even exclusive somatic basis in the sense of non-experientially determined constitutional biochemical factors. The psychiatrist interested in and experienced in exploring and treating personal and interpersonal problems as a basis of ill-health may become more involved in other disorders whose manifestations are somatic and in which the importance of psychological factors are hidden by the patient's presenting mechanism of denial. In general hospitals such patients are readily available to the psy- chiatrist and, as Brough \& Fleminger (1965) have pointed out, are inevitably one of his special concerns. In this role, in my experience, he can expect goodwill and interest from a majority of his medical colleagues, junior medical staff and nursing staff (indeed the medical ward sister can sometimes be a perceptive and useful witness to the patient's behaviour and his relationships with other patients and with relatives). The medical social workers will often welcome the opportunity of working with him. In such a position he can expect to provide specialized help to some patients and indulge any research interest he has. I suspect that it is important for him to separate off any too optimistic a view of the help he can offer from whatever are his views concerning that often indistinct field, the psychopathology in any one patient. Ultimately his position will be dictated, apart from his own inclination and interest, by whether he is seen to be and found to be of value to his medical colleagues. Such help, as already indicated, will be in the form of availability of adjacent psychiatric beds for the purposes of transfer of patients sometimes continuing with joint psychiatric and medical management; alternatively the provision of acceptable psychological understanding of and/or clear value of the psychological treatment of patients seen and treated in the physician's beds or else back in the community jointly cared for by their general practitoner, the physician and the psychiatrist.

\section{Acknowledgments}

The author is particularly indebted to and grateful to Dr J. D. N. Nabarro for his help and encouragement with this study and to Miss E. Russell for her secretarial assistance and help with the records.

\section{References}

Bridges, P.K., Koller, K.M. \& Wheeler, T.K. (1966) Psychiatric referrals in a general hospital. Acta psychiat. Scand. 42, 171.

Brough, D.I. \& Fleminger, J.J. (1965) Psychiatric referral of medical and surgical out-patients. Guy's Hosp. Rep. 114, 291.

BRYSON, E. (1945) The psychosomatic approach in gynaecological practice. Practitioner, 155, 378.

CrISP, A.H. (1967) Anorexia nervosa: 'feeding disorder', 'nervous malnutrition' or 'weight phobia'. Hosp. Med. $1,713$.

Culpan, R., Davies, B. \& Oppenheim, A. (1960) Incidence of psychiatric illness among hospital out-patients. Brit. med. J. i, 855.

DAvies, B. (1964) Psychiatric illness at general hospital clinics. Postgrad. med. J. 40, 15.

ENGEL, G.L. (1967) Medical education and the psychosomatic approach-A report on the Rochester experience 19461966. J. psychosom. Res. $11,77$.

Fleminger, J.J. \& MAllett, B.L. (1962) Psychiatric referrals from medical and surgical wards. J. ment. Sci. 108, 183. 
Herridge, C. (1960) Physical disorders in psychiatric illness. Lancet, ii, 949.

Hill, D. \& Hinton, J. (1966) A programme of undergraduate education in psychiatry. Brit. med. J. ii, 460.

JacoBs, M. \& Ritchie Russell, W. (1961) 'Functional' disorders. A follow-up study of out-patient diagnosis. Brit. med. J. ii, 346.

Kaufman, M.R. (1965) (Ed.) The Psychiatric Unit in a General Hospital: Its Current and Future Role. Internat. Univ. Press.

KENYON, F.E. \& RutTER, M.L. (1963) The psychiatrist and the general hospital. Comp. Psychiat. 4, 80.

McKeOWN, J. (1966) Hospital planning. Lancet, i, 869.
Maclay, I. (1965) The functional medical out-patient. Brit. J. Psychiat. 111, 34.

Memorandum on amalgamation of psychiatric with general hospital groups. (1967) Brit. J. Psychiat. 113, 235.

Priest, W. M. (1962) A thousand out-patients. Lancet, ii, 1043.

Richmond, J.B. (1965) In: The Psychiatric Unit in a General Hospital: Its Current and Future Role (Ed. by M. R. Kaufman), p. 432. Internat. Univ. Press.

SloANe, R.B. (1963) Psychotherapy in a general hospital. Proceedings of the 5th International Congress of Psychotherapy, Vol. 3, Part III, pp. 81-94. Karger, Basel. 